2013

\title{
Recruitment of the Intracellular Ca2+ By Ultrashort Electric Stimuli: The Impact of Pulse Duration
}

Iurii Semenov

Old Dominion University, isemenov@odu.edu

Shu Xiao

Old Dominion University, sxiao@odu.edu

Olga N. Pakhomova

Old Dominion University, opakhomo@odu.edu

Andrei G. Pakhomov

Old Dominion University, apakhomo@odu.edu

Follow this and additional works at: https://digitalcommons.odu.edu/bioelectrics_pubs

Part of the Biochemistry Commons, Biomedical Commons, and the Cell Biology Commons

\section{Repository Citation}

Semenov, Iurii; Xiao, Shu; Pakhomova, Olga N.; and Pakhomov, Andrei G., "Recruitment of the Intracellular Ca2+ By Ultrashort Electric Stimuli: The Impact of Pulse Duration" (2013). Bioelectrics Publications. 203.

https://digitalcommons.odu.edu/bioelectrics_pubs/203

\section{Original Publication Citation}

Semenov, I., Xiao, S., Pakhomova, O. N., \& Pakhomov, A. G. (2013). Recruitment of the intracellular $\mathrm{Ca}^{2+}$ by ultrashort electric stimuli: The impact of pulse duration. Cell Calcium, 54(3), 145-150. doi:10.1016/j.ceca.2013.05.008 


\title{
Recruitment of the intracellular $\mathrm{Ca}^{2+}$ by ultrashort electric stimuli: the impact of pulse duration
}

\author{
lurii Semenova, ${ }^{\star}$, Shu Xiao ${ }^{a, b}$, Olga N. Pakhomovaa ${ }^{a}$, and Andrei G. Pakhomova \\ aFrank Reidy Research Center for Bioelectrics, Old Dominion University, Norfolk, VA \\ bDept. of Electrical and Computer Engineering, Old Dominion University, Norfolk, VA, USA
}

\section{Abstract}

Nanosecond-duration electric stimuli are distinguished by the ability to permeabilize intracellular membranes and recruit $\mathrm{Ca}^{2+}$ from intracellular stores. We quantified this effect in non-excitable cells (CHO) using ratiometric $\mathrm{Ca}^{2+}$ imaging with Fura-2. In a $\mathrm{Ca}^{2+}$-free medium, 10-, 60-, and 300 -ns stimuli evoked $\mathrm{Ca}^{2+}$ transients by mobilization of $\mathrm{Ca}^{2+}$ from the endoplasmic reticulum. With $2 \mathrm{mM}$ external $\mathrm{Ca}^{2+}$, the transients included both extra- and intracellular components. The recruitment of intracellular $\mathrm{Ca}^{2+}$ increased as the stimulus duration decreased. At the threshold of 200-300 nM, the transients were amplified by calcium-induced calcium release. We conclude that nanosecond stimuli mimic $\mathrm{Ca}^{2+}$ signaling while bypassing the usual receptor- and channelsmediated cascades. The recruitment of the intracellular $\mathrm{Ca}^{2+}$ can be controlled by the duration of the stimulus.

\section{Keywords}

Nanosecond pulses; Electroporation; Electropermeabilization; Calcium signaling; Electric field

\section{Introduction}

High-intensity electric pulses of nanosecond duration (nsEP), also often called nanosecond pulsed electric fields, were originally introduced to biology as a means to electroporate intracellular membranous structures [1-3]. During the last decade, a number of unique nsEP bioeffects and applications have been reported, although mechanisms of nsEP effects on cells have not been fully understood.

In the case of traditional electroporation with micro- and millisecond duration pulses, charging of the cell plasma membrane (PM) compensates the external electric field and protects the intracellular structures. However, calculations showed that nsEP can charge smaller intracellular structures up to the electroporation threshold faster than it would take for PM to charge and protect them [2-4]. Indeed, independent experimental studies

\footnotetext{
(C) 2013 Elsevier Ltd. All rights reserved.

*Corresponding author: Iurii Semenov, Frank Reidy Research Center for Bioelectrics, Old Dominion University, 4211 Monarch Way, Suite 319, Norfolk, VA 23508, 757-683-2234, 210-254-6324 (cell), 757-451-1010 (fax), iurii.semenov@ gmail.com.

Conflict of interest

All co-authors of the following manuscript named "Recruitment of the intracellular $\mathrm{Ca}^{2+}$ by ultrashort electric stimuli: the impact of pulse duration" declare no conflict of interest.

Publisher's Disclaimer: This is a PDF file of an unedited manuscript that has been accepted for publication. As a service to our customers we are providing this early version of the manuscript. The manuscript will undergo copyediting, typesetting, and review of the resulting proof before it is published in its final citable form. Please note that during the production process errors may be discovered which could affect the content, and all legal disclaimers that apply to the journal pertain.
} 
demonstrated the electroporation of mitochondria, endoplasmic reticulum (ER), and vacuoles with nsEP [2-5]. Although these studies claimed the lack of concurrent PM disruption by nsEP, the methods employed to compare the poration of the intracellular structures and of the PM were different. For instance, White et al. [5] used $\mathrm{Ca}^{2+}$ efflux from the ER to assess the poration of ER and the uptake of propidium iodide (PI) to assess the poration of PM. In a study by Tekle et al. [4], permeabilization of vacuoles by nsEP was detected by the efflux of Calcein dye, whereas the PM integrity was judged by the uptake of the ethidium homodimer. In view of later findings of high selectivity of nsEP-formed membrane pores $[6,7]$, the above data can no longer be taken as a proof of selective intracellular poration.

Further studies focused specifically on the PM have found that it is certainly not exempt from the poration by nsEP. Exposures to nsEP increased the PM electrical conductance [6, $8,9]$ and the uptake of small dyes [7, 10], ions [10-13], and water [14]. The formation of small membrane pores ("nanopores"), concurrently in both PM and intracellular membranes was supported by advanced numerical models $[15,16]$. The facts that more intense nsEP treatments can actually cause PI uptake $[6,7,17]$ and that long pulses can cause intracellular effects once the PM is compromised [18] have further blurred the difference between the traditional electroporation and nsEP.

As of today, it has not been experimentally demonstrated that reducing the width of electric pulses increases their intracellular effect. In addition to the arbitrary and "asymmetrical" choice of the electroporation markers for PM and intracellular poration, the critical question was the choice of comparable parameters for short and long electric stimuli. For example, a $20-\mathrm{Hz}$ train of 100 pulses of 50ns duration at $6.7 \mathrm{kV} / \mathrm{cm}$ was arbitrarily compared to a single $400-\mu$ s pulse at $1.4 \mathrm{kV} / \mathrm{cm}$ [4]. Such comparisons are inconclusive and misleading without proper scaling of parameters from nsEP to longer pulses.

The goal of the present study was to test, under stringent conditions, the theoretical predictions that the intracellular effects of nsEP increase when the pulse duration is decreased. The increase in cytosolic $\mathrm{Ca}^{2+}$ caused by nsEP was chosen as a unique and universal endpoint for this study. In the previous study [11], we have shown that in Chinese hamster ovary $(\mathrm{CHO})$ cells, this increase can occur by either $\mathrm{Ca}^{2+}$ entry from the outside (PM poration) or $\mathrm{Ca}^{2+}$ discharge from the ER (intracellular poration). This relatively simple cell model was chosen for the lack of voltage-gated PM channels and ryanodine receptors, which helps the interpretation of nsEP effects.

In addition, the use of $\mathrm{Ca}^{2+}$ as a criterion of both PM and ER poration has made it possible to utilize ratiometric imaging [19] and measure $\mathrm{Ca}^{2+}$ changes in actual concentration units rather than as fold changes or arbitrary units.

Finally, for a comparison between 10-, 60-, and 300-ns stimuli, we employed a concept of isoeffective treatments. $\mathrm{Ca}^{2+}$ transients were always evoked by a single stimulus. First, in the presence of $2 \mathrm{mM}$ external $\mathrm{Ca}^{2+}$, the intensities of the stimuli were empirically adjusted in order to produce $\mathrm{Ca}^{2+}$ responses of similar amplitude by the stimuli of different duration. Next, these isoeffective intensities of 10-, 60-, and 300-ns pulses were tested in a $\mathrm{Ca}^{2+}$-free medium, in order to compare their potency to recruit the intracellular $\mathrm{Ca}^{2+}$. In agreement with theoretical predictions, we established that the intracellular effect was the highest for $10-n s$ pulses and minimal for 300-ns pulses. 


\section{Materials and Methods}

\subsection{Cell culture}

Experiments were performed in $\mathrm{CHO}-\mathrm{K} 1$ cells obtained from the American Type Culture Collection (ATCC, Manassas, VA). Cells were propagated at $37{ }^{\circ} \mathrm{C}$ with $5 \% \mathrm{CO}_{2}$ in air in Ham's F12K medium supplemented with $10 \%$ fetal bovine serum, 100 I.U./ml penicillin, and $0.1 \mu \mathrm{g} / \mathrm{ml}$ streptomycin. The medium and its components were purchased from Mediatech Cellgro (Herdon, VA) except for the serum (Atlanta Biologicals, Norcross, GA).

\subsection{Calcium imaging}

The detailed procedures employed for loading cells with Fura-2, dye calibration, and time lapse fluorescence imaging were reported elsewhere [11]. Cells were transferred onto glass coverslips 12-24 h prior to experiments. After loading with the dye, cells were placed in a glass-bottomed perfusion chamber mounted on an IX71 microscope (Olympus America, Center Valley, PA).

Cells were continually perfused with a physiological solution containing (in $\mathrm{mM}$ ): 140 $\mathrm{NaCl}, 5.4 \mathrm{KCl}, 1.5 \mathrm{MgCl}_{2}, 2 \mathrm{CaCl}_{2}, 10$ glucose, and 10 HEPES (pH 7.3, 300 mOsm/kg). For $\mathrm{Ca}^{2+}$-free conditions, $\mathrm{CaCl}_{2}$ was replaced with $2 \mathrm{mM}$ Na-EGTA.

A fast wavelength switcher Lambda DG4 (Sutter Instruments, Novato, CA) was employed for dye excitation alternatively at 340 and $380 \mathrm{~nm}$. Emission was measured at $510 \mathrm{~nm}$ with a C9100-02 EM CCD camera (Hamamatsu Photonics, Japan). The cytosolic free $\mathrm{Ca}^{2+}$ concentration $\left(\left[\mathrm{Ca}^{2+}\right]_{\mathrm{i}}\right)$ was calculated from Fura-2 emission ratio with a help of Metafluor v.7.5 software (Molecular Devices, Sunnyvale, CA). $\mathrm{Ca}^{2+}$ measurements typically began one minute prior to nsEP exposure.

Fura-2 pentapotassium salt, Fura-2/AM, calcium calibration buffer kit, and Pluronic F-127 (20\% solution in DMSO) were purchased from Life Technologies (Grand Island, NY). Thapsigargin (TG), a specific and irreversible blocker of $\mathrm{Ca}^{2+}$-ATPase of the ER, was purchased from Tocris Bioscience (Minneapolis, MN). Other chemicals were from SigmaAldrich (St. Louis, MO).

\subsection{NsEP exposure and simulation of the local electric field}

Nearly rectangular pulses of 10-, 60-, and 300-ns duration were produced by dedicated pulse generators. 300-ns pulses (up to $450 \mathrm{~V}$ ) were generated in a transmission line-type circuit, by closing a MOSFET switch upon delivery of a TTL trigger pulse [7, 8]. To produce 60 -ns pulses (up to $800 \mathrm{~V}$ output), we employed a Blumlein line-type circuit [11]. 10-ns pulses of up to $20 \mathrm{kV}$ amplitude were produced by a model FPG 20-1NM pulse generator (FID GmbH, Burbach, Germany).

Pulses were delivered to selected cells on coverslip with a pair of tungsten rod electrodes [7, $11,20]$. The exact pulse shapes and amplitudes were captured and measured with a TDS 3052 oscilloscope (Tektronix, Beaverton, OR). The $10 \%$ to $90 \%$ rise time was $0.6 \mathrm{~ns}$ for 10 ns pulses and 4.5 ns for both 60 - and 300-ns pulses. Representative pulse waveforms are shown in Fig. 1.

NsEP were triggered externally and synchronized with image acquisition by a TTL pulse protocol using Digidata 1440A board and Clampex v. 10.2 software (Molecular Devices). In most experiments, $4-8$ cells were stimulated together with nsEP. We did not observe any systematic differences in responses to nsEP that could be attributed to cell size, shape, or the number and configuration of cells in the group. Any group of cells was exposed only once. For statistical analysis, the experiments were repeated 3-10 times in different cell groups 
and on different coverslips. Different treatment conditions were alternated in a random manner.

The E-field at the cell location between the electrodes was determined as reported previously [7, 11,20], by 3D simulations with a finite element Maxwell equations solver Amaze 3D (Field Precision, Albuquerque, NM).

\subsection{Data analysis}

Origin 8.0 (OriginLab Corporation, Northampton, MA) was utilized for smoothing and differentiation of traces, and for statistical analysis. $\mathrm{Ca}^{2+}$ transients shown in Figs. 2-5 have been averaged from 15-50 cells. The data for statistical analysis were measured from the original traces before averaging. The results were expressed as the mean \pm s.e. and deemed significant at $p<0.05$ (Student's $t$-test for unpaired data).

\section{Results and discussion}

\subsection{PM and ER permeabilization by $10-, 60-$, and $300-n s$ pulses}

As we showed earlier [11], nsEP-induced $\mathrm{Ca}^{2+}$ transients in $\mathrm{CHO}$ cells may involve $\mathrm{Ca}^{2+}$ entry through PM, $\mathrm{Ca}^{2+}$ discharge from the intracellular lumens, and active amplification of the response by calcium-induced calcium release (CICR). We also demonstrated that these mechanisms can be separated by manipulation of the bath $\mathrm{Ca}^{2+}$ and using pharmacological blockers. The ER was the only significant source of the intracellular $\mathrm{Ca}^{2+}$ in $\mathrm{CHO}$ cells.

In the presence of $2 \mathrm{mM}$ external $\mathrm{Ca}^{2+}$, we tuned the intensity of 10- and 60-ns stimuli to evoke $\mathrm{Ca}^{2+}$ transients of similar amplitude. Not surprisingly, shorter stimuli had to be applied at higher intensities to evoke the same $\mathrm{Ca}^{2+}$ response (the difference for 10- and 60ns stimuli was almost 10 -fold, Fig. 2, red traces). These $\mathrm{Ca}^{2+}$ transients were produced by a combination of $\mathrm{Ca}^{2+}$ uptake via PM and $\mathrm{Ca}^{2+}$ discharge from the ER, either without activation of CICR (top panels) or with it (bottom panels). The fraction of the response due to the recruitment of $\mathrm{Ca}^{2+}$ from ER was estimated by the removal of the bath $\mathrm{Ca}^{2+}$ and applying the same 10- and 60-ns stimuli (blue traces). As seen in Fig. 2, for the entire range of tested nsEP intensities, the contribution of $\mathrm{Ca}^{2+}$ from the intracellular stores was profoundly greater for 10 -ns stimuli than for 60 -ns stimuli. Of note, different shapes of transients induced by the most intense10- and 60-ns EP in the presence of $\mathrm{Ca}^{2+}$ (red traces in the bottom panels) could also be a result of greater ER engagement by 10-ns pulses.

Fig. 3 compares $\mathrm{Ca}^{2+}$ responses to 60- and 300-ns stimuli. Fig. 3A shows $\mathrm{Ca}^{2+}$ traces evoked by 60 -ns stimuli of the highest intensity allowed by our pulse generator. The response peaked at $2 \mu \mathrm{M}$ in the presence of $2 \mathrm{mM}$ external $\mathrm{Ca}^{2+}$ (red trace) and dropped to $0.7 \mu \mathrm{M}$ in the absence of it (blue trace). The red trace in Fig. $3 \mathrm{~B}$ shows $\mathrm{Ca}^{2+}$ transient of the same amplitude as in (A), but evoked by a 300-ns stimulus. In contrast to the data in panel A, the removal of external $\mathrm{Ca}^{2+}$ completely abolished the response to 300-ns stimuli. Likewise, 300-ns stimuli did not recruit any intracellular $\mathrm{Ca}^{2+}$ at lower stimulus intensities corresponding to the $\mathrm{Ca}^{2+}$ transients in Fig. 2 (data not shown). However, further increase of the stimulus intensity beyond the level that could be matched by shorter pulses has made it possible to evoke $\mathrm{Ca}^{2+}$ discharge from the ER even by 300-pulses (Fig. 3C).

The graphs in Fig. 4A compare the efficiency of 10-, 60- and 300-ns stimuli to produce $\mathrm{Ca}^{2+}$ transients in the presence of external $\mathrm{Ca}^{2+}$ (red symbols) and in the absence of it (blue symbols). Increasing the stimulus intensity increased the amplitude of the response in a characteristically non-linear fashion, reflecting the amplification of the response by CICR once the threshold of 200-300 $\mathrm{nM}$ was exceeded [11]. Within the entire tested range of stimulus intensities and irrespective of the emergence of CICR, $\mathrm{Ca}^{2+}$ recruitment from the 
ER contributed most to the effect of 10-ns stimuli and the least to the effect of 300-ns stimuli.

In the summary graph (Fig. 4B), the stimulus intensity was taken out of the picture. The amplitude of the $\mathrm{Ca}^{2+}$ response for different nsEP stimuli under $\mathrm{Ca}^{2+}$-free conditions was plotted against the response in the presence of $2 \mathrm{mM} \mathrm{Ca}^{2+}$. The characteristic upward bend of the curves corresponds to the threshold of CICR under $\mathrm{Ca}^{2+}$-free conditions. Once again, these graphs show that under all tested conditions 10-ns pulses were the most efficient in recruiting $\mathrm{Ca}^{2+}$ from the intracellular stores.

\subsection{The rate of $\mathrm{Ca}^{2+}$ rise as an index of ER involvement}

In the above experiments, individual cells within a microscope field of view were selected as "regions of interest" for $\mathrm{Ca}^{2+}$ measurements. $\left[\mathrm{Ca}^{2+}\right]_{\mathrm{i}}$ as measured by this approach was averaged over the entire cell volume. As noted by studies of $\mathrm{Ca}^{2+}$ dynamics in myocytes $[21,22], \mathrm{Ca}^{2+}$ entry through the PM occurs in a fraction of the cell volume, followed by $\mathrm{Ca}^{2+}$ diffusion; these factors increase the apparent duration of $\mathrm{Ca}^{2+}$ rise when its concentration is averaged over the cell volume. In contrast, membranes of the ER are widely distributed inside the cell, so $\mathrm{Ca}^{2+}$ discharge from the ER spreads throughout the cell volume faster than $\mathrm{Ca}^{2+}$ that enters through the PM. If this rule holds true for $\mathrm{CHO}$ cells, the increased rate of nsEP-induced $\mathrm{Ca}^{2+}$ rise can serve as another manifestation of the ER involvement in the response.

To produce a $\mathrm{Ca}^{2+}$ transient solely by $\mathrm{Ca}^{2+}$ entrance through the PM, the $\mathrm{ER} \mathrm{Ca}^{2+}$ store was fully depleted by a 30-min preincubation with $100 \mathrm{nM}$ thapsigargin [11]. For comparison, transients caused solely by $\mathrm{Ca}^{2+}$ discharge from the ER were evoked in a $\mathrm{Ca}^{2+}$-free medium. To inhibit possible amplification of the response by CIRC, we blocked inositol-1,4,5trisphosphate receptors of the ER with $50 \mu \mathrm{M}$ of 2-APB (2-aminoethoxydiphenyl borate) [11].

Fig. 5, A-C shows that under the above conditions the rate of $\mathrm{Ca}^{2+}$ rise was indeed dependent on the source of $\mathrm{Ca}^{2+}$ : The discharge from the ER resulted in a shorter rise time than $\mathrm{Ca}^{2+}$ uptake through the PM (Fig. 5A). In the latter case, the rise time did not depend on whether the nsEP duration was 10 or $60 \mathrm{~ns}$.

The role of the $\mathrm{Ca}^{2+}$ source can be appreciated better by differentiation of the original traces to measure the rate of $\mathrm{Ca}^{2+}$ rise (Fig. 5B). Of note, the percentile filter of Origin 8 that was employed to improve the signal-to-noise ratio has also decreased the peak amplitude and produced an artifact of the premature onset of the response. Hence the traces in Fig 5B serve for illustration purpose only, whereas the quantitative data measured from undistorted traces are provided in Fig. 5C. Indeed, the cell volume-average rate of $\mathrm{Ca}^{2+}$ increase was more than twofold higher when $\mathrm{Ca}^{2+}$ came from the ER as compared to its entry through the PM. Thus, the rate of $\mathrm{Ca}^{2+}$ rise depended on the source of $\mathrm{Ca}^{2+}$ but not on the nsEP duration, and therefore could be utilized to distinguish between PM and ER poration.

These data have laid the ground for rate comparison of $\mathrm{Ca}^{2+}$ transients evoked by $10-$ and 60-ns stimuli in the presence of $2 \mathrm{mM} \mathrm{Ca}^{2+}$ (Fig. 5D). As noted above, such transients involve both $\mathrm{Ca}^{2+}$ entry from the outside and its discharge from the ER. For different response amplitudes (corresponding to the actual traces in Fig 2), the rate of $\mathrm{Ca}^{2+}$ rise was always higher for 10-ns pulses, thus indicating greater contribution of the ER to the overall response.

Thus, two different approaches have identified 10-ns stimuli as the most efficient to recruit the intracellular $\mathrm{Ca}^{2+}$. These experimental data are in agreement with theoretical predictions 
that nsEP have the ability to electroporate intracellular structures, and that this ability is higher for shorter pulses. It should be noted that the efficiency was evaluated relative to the ability of the same pulse to electroporate the PM, irrespective of the fact that the required intensity was much higher for shorter nsEP.

The deliberate adjustment of the nsEP duration opens an avenue for accurate control of $\mathrm{Ca}^{2+}$ signaling, by varying the extent of the intracellular $\mathrm{Ca}^{2+}$ recruitment. $\mathrm{Ca}^{2+}$ transients evoked by nsEP seem to be "interpreted" by cells as natural $\mathrm{Ca}^{2+}$ signals and further amplified by CICR, thus mimicking $\mathrm{Ca}^{2+}$ signals that originate from the activation of PM receptors or ion channels. Stimulation of cells by nsEP has the potential to develop into a unique tool for precise but non-chemical activation of $\mathrm{Ca}^{2+}$ signaling mechanisms.

\section{Acknowledgments}

The study was supported by R01CA125482 from the National Cancer Institute and R01GM088303 from the National Institute of General Medical Sciences.

\section{References}

1. Deng, J.; Stark, RH.; Schoenbach, KH. A nanosecond pulse generator for intracellular electromanipulation. Twenty-Fourth Intern. Power Modulator Symp; Norfolk, VA. 2000. p. 47-50.

2. Schoenbach KH, Beebe SJ, Buescher ES. Intracellular effect of ultrashort electrical pulses. Bioelectromagnetics. 2001; 22:440-448. [PubMed: 11536285]

3. Schoenbach KS, Hargrave B, Joshi RP, Kolb J, Osgood C, Nuccitelli R, Pakhomov AG, Swanson J, Stacey M, White JA, Xiao S, Zhang J, Beebe SJ, Blackmore PF, Buescher ES. Bioelectric Effects of Nanosecond Pulses. IEEE Transactions on Dielectrics and Electrical Insulation. 2007; 14:10881109.

4. Tekle E, Oubrahim H, Dzekunov SM, Kolb JF, Schoenbach KH, Chock PB. Selective Field Effects on Intracellular Vacuoles and Vesicle Membranes with Nanosecond Electric Pulses. Biophysical Journal. 2005; 89:274-284. [PubMed: 15821165]

5. White JA, Blackmore PF, Schoenbach KH, Beebe SJ. Stimulation of capacitative calcium entry in HL-60 cells by nanosecond pulsed electric fields. J Biol Chem. 2004; 279:22964-22972. [PubMed: 15026420]

6. Pakhomov, AG.; Pakhomova, ON. Nanopores: A distinct transmembrane passageway in electroporated cells. In: Pakhomov, AG.; Miklavcic, D.; Markov, MS., editors. Advanced Electroporation Techniques in Biology in Medicine. CRC Press; Boca Raton: 2010. p. 178-194.

7. Bowman AM, Nesin OM, Pakhomova ON, Pakhomov AG. Analysis of plasma membrane integrity by fluorescent detection of Tl(+) uptake. J Membr Biol. 2010; 236:15-26. [PubMed: 20623351]

8. Pakhomov AG, Bowman AM, Ibey BL, Andre FM, Pakhomova ON, Schoenbach KH. Lipid nanopores can form a stable, ion channel-like conduction pathway in cell membrane. Biochem Biophys Res Commun. 2009; 385:181-186. [PubMed: 19450553]

9. Pakhomov AG, Kolb JF, White JA, Joshi RP, Xiao S, Schoenbach KH. Long-lasting plasma membrane permeabilization in mammalian cells by nanosecond pulsed electric field (nsPEF). Bioelectromagnetics. 2007; 28:655-663. [PubMed: 17654532]

10. Vernier PT, Sun Y, Gundersen MA. Nanoelectropulse-driven membrane perturbation and small molecule permeabilization. BMC Cell Biol. 2006; 7:37. [PubMed: 17052354]

11. Semenov I, Xiao S, Pakhomov AG. Primary pathways of intracellular $\mathrm{Ca}(2+)$ mobilization by nanosecond pulsed electric field. Biochim Biophys Acta. 2013; 1828:981-989. [PubMed: 23220180]

12. Wang S, Chen J, Chen MT, Vernier PT, Gundersen MA, Valderrabano M. Cardiac myocyte excitation by ultrashort high-field pulses. Biophys J. 2009; 96:1640-1648. [PubMed: 19217879]

13. Beier HT, Roth CC, Tolstykh GP, Ibey BL. Resolving the spatial kinetics of electric pulse-induced ion release. Biochem Biophys Res Commun. 2012; 423:863-866. [PubMed: 22713455] 
14. Nesin OM, Pakhomova ON, Xiao S, Pakhomov AG. Manipulation of cell volume and membrane pore comparison following single cell permeabilization with 60- and 600-ns electric pulses. Biochim Biophys Acta. 2011; 1808:792-801. [PubMed: 21182825]

15. Gowrishankar TR, Weaver JC. Electrical behavior and pore accumulation in a multicellular model for conventional and supra-electroporation. Biochem Biophys Res Commun. 2006; 349:643-653. [PubMed: 16959217]

16. Kotnik T, Miklavcic D. Theoretical evaluation of voltage inducement on internal membranes of biological cells exposed to electric fields. Biophys J. 2006; 90:480-491. [PubMed: 16239325]

17. Napotnik TB, Rebersek M, Kotnik T, Lebrasseur E, Cabodevila G, Miklavcic D. Electropermeabilization of endocytotic vesicles in B16 F1 mouse melanoma cells. Med Biol Eng Comput. 2010; 48:407-413. [PubMed: 20361267]

18. Esser AT, Smith KC, Gowrishankar TR, Vasilkoski Z, Weaver JC. Mechanisms for the Intracellular Manipulation of Organelles by Conventional Electroporation. Biophysical Journal. 2010; 98:2506-2514. [PubMed: 20513394]

19. Grynkiewicz G, Poenie M, Tsien RY. A new generation of Ca2+ indicators with greatly improved fluorescence properties. J Biol Chem. 1985; 260:3440-3450. [PubMed: 3838314]

20. Pakhomova ON, Gregory BW, Khorokhorina VA, Bowman AM, Xiao S, Pakhomov AG. Electroporation-induced electrosensitization. PloS one. 2011; 6:e17100. [PubMed: 21347394]

21. Michailova A, DelPrincipe F, Egger M, Niggli E. Spatiotemporal features of Ca2+ buffering and diffusion in atrial cardiac myocytes with inhibited sarcoplasmic reticulum. Biophys J. 2002; 83:3134-3151. [PubMed: 12496084]

22. Thul R, Coombes S, Roderick HL, Bootman MD. Subcellular calcium dynamics in a whole-cell model of an atrial myocyte. Proceedings of the National Academy of Sciences of the United States of America. 2012; 109:2150-2155. [PubMed: 22308396] 


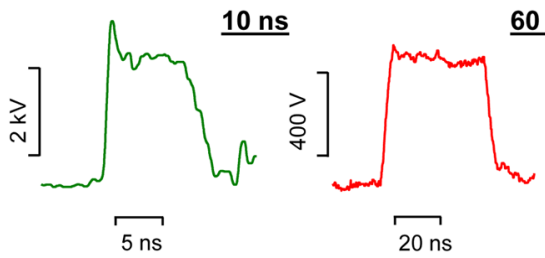

$60 \mathrm{~ns}$
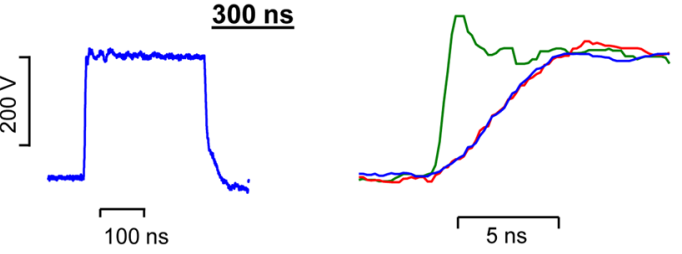

Fig. 1.

Sample waveforms and comparison of rise times for 10-, 60-, and 300-ns stimuli 

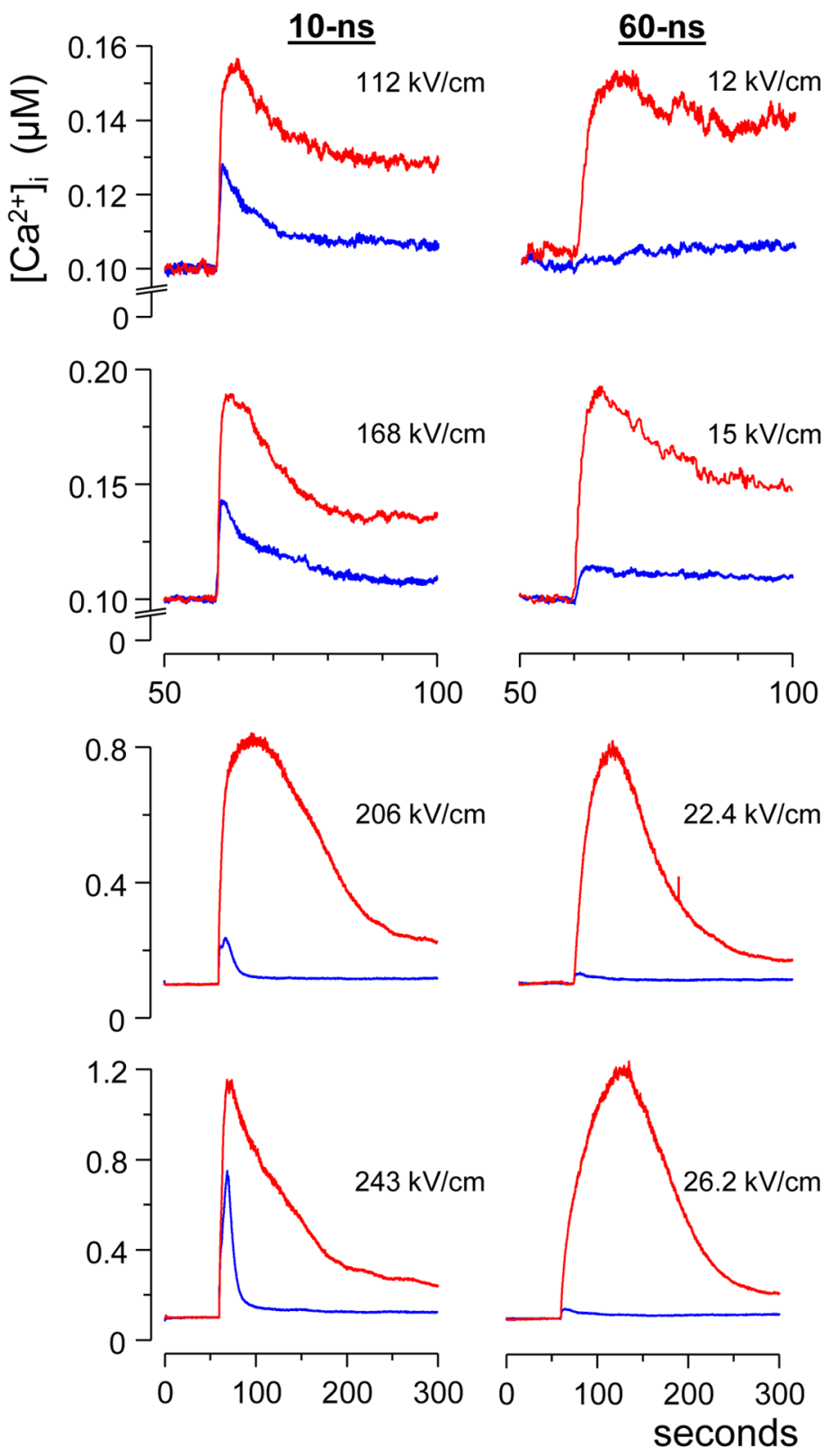

Fig. 2.

Comparison of $\mathrm{Ca}^{2+}$ transients evoked by 10 - and 60 -ns stimuli. Each trace is the average from at least 15 cells; nsEP was applied at $60 \mathrm{~s}$. The nsEP intensity $(\mathrm{kV} / \mathrm{cm})$ was adjusted to evoke similar responses by 10 - and 60 -ns stimuli in the presence of $2 \mathrm{mM}$ external $\mathrm{Ca}^{2+}(\mathrm{red}$ traces). The fraction of $\mathrm{Ca}^{2+}$ coming from the endoplasmic reticulum (ER) was assessed by applying the same nsEP in the absence of external $\mathrm{Ca}^{2+}$ (blue traces). Note that 10 -ns pulses consistently evoked higher ER response than 60-ns ones. See text for further details. 

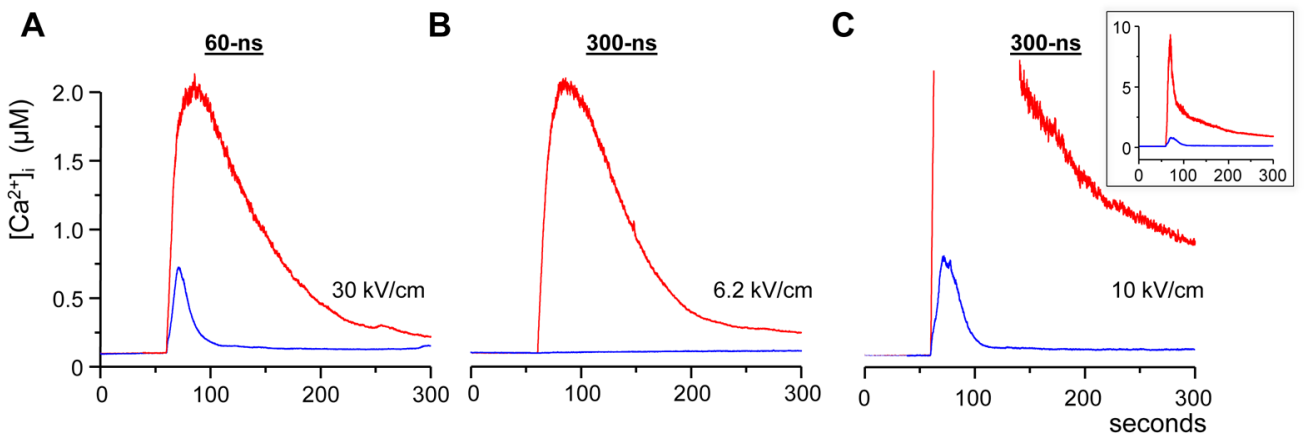

Fig. 3.

Comparison of $\mathrm{Ca}^{2+}$ transients evoked by 60 - and 300-ns stimuli. (A) The transients evoked by a maximum intensity $60-n s$ pulse in the presence and absence of $2 \mathrm{mM}$ external $\mathrm{Ca}^{2+}$ (hereinafter, shown by red and blue traces, respectively). (B) The intensity of a 300-ns pulse was adjusted, in the presence of $2 \mathrm{mM} \mathrm{Ca}^{2+}$, to evoke a similar $\mathrm{Ca}^{2+}$ response as in panel (A). The removal of external $\mathrm{Ca}^{2+}$ completely eliminated the response to the $300-n \mathrm{~s}$ pulse. (C) In principle, 300-ns stimuli were capable of recruiting the intracellular $\mathrm{Ca}^{2+}$, but only at a high intensity that could not be matched with shorter pulses. The inset shows the full amplitude of the response. 

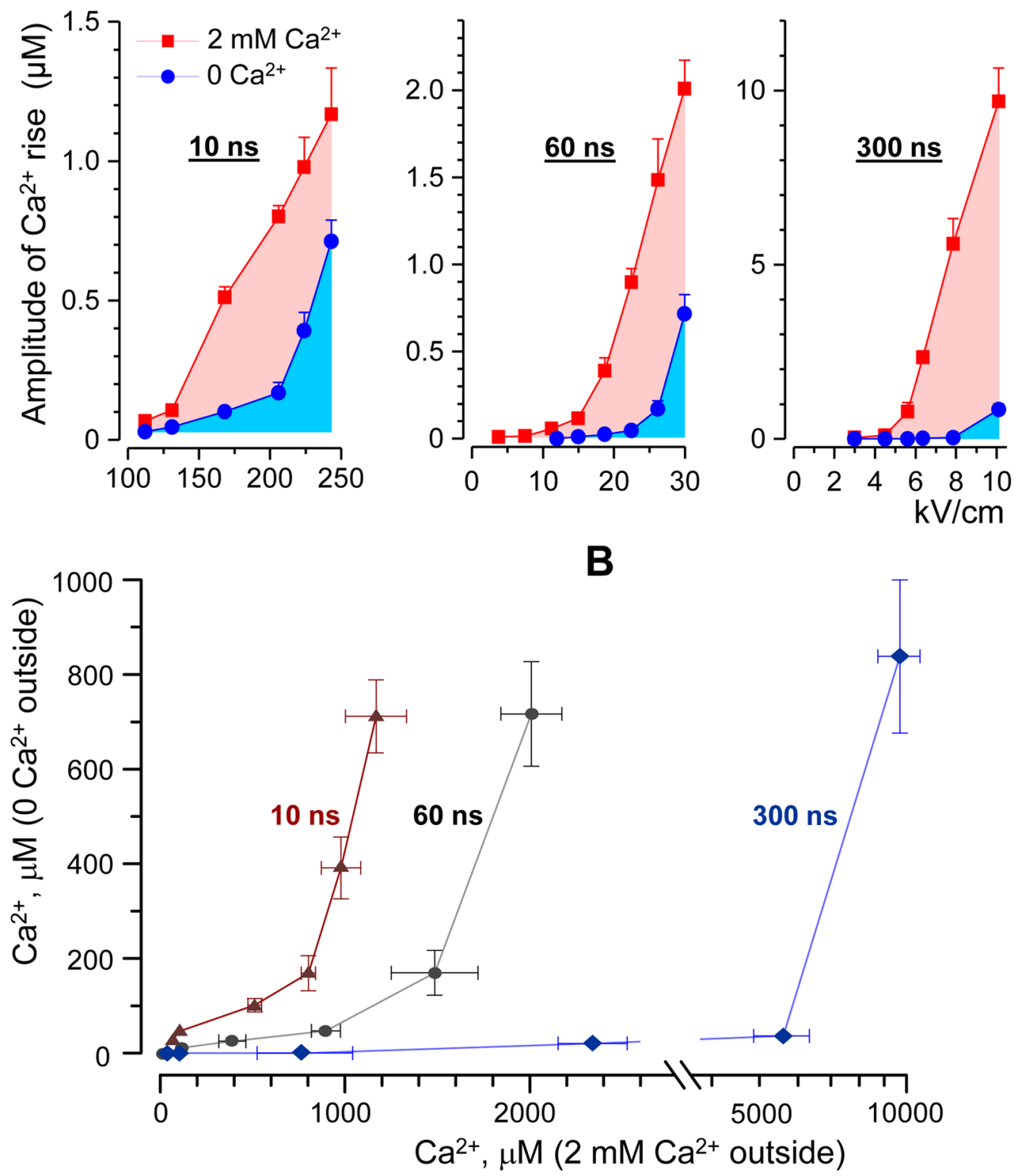

Fig. 4.

Shorter nsEP are more efficient in recruiting $\mathrm{Ca}^{2+}$ from the intracellular stores. (A) The peak amplitude of $\mathrm{Ca}^{2+}$ transients evoked by 10-, 60- and 300-ns stimuli was plotted against the stimulus intensity $(\mathrm{kV} / \mathrm{cm})$, mean $+/-$ s.e.; $\mathrm{n} \geq 15$. Cells were stimulated in the presence of 2 $\mathrm{mM}$ external $\mathrm{Ca}^{2+}$ (red) or its absence (blue). In the latter case, the endoplasmic reticulum was the only significant source of $\mathrm{Ca}^{2+}$. Note the increasing contribution of the intracellular $\mathrm{Ca}^{2+}$ as the stimulus duration is decreased. (B) The amplitude of $\mathrm{Ca}^{2+}$ response due to the discharge from the intracellular stores was plotted against the amplitude of the compound response (due to both the uptake from the outside and the discharge from the stores). The data shown are from the same experiments as in panel (A). For any amplitude of the compound response, shorter stimuli evoked more intracellular $\mathrm{Ca}^{2+}$ discharge. 

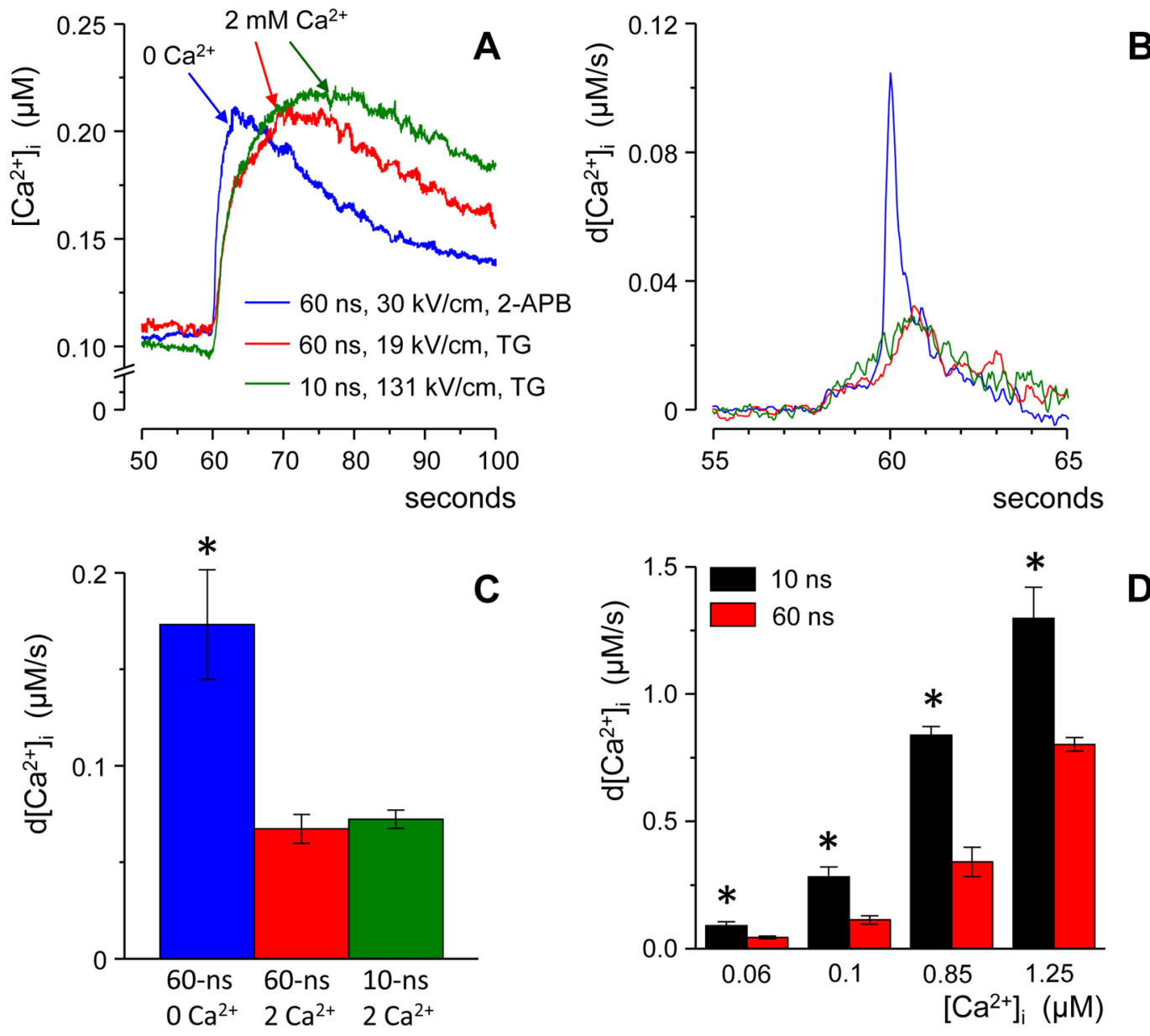

Fig. 5.

$\mathrm{Ca}^{2+}$ discharge from the endoplasmic reticulum (ER) is manifested by a faster rise of the nsEP-induced $\mathrm{Ca}^{2+}$ transient. (A) Traces of $\mathrm{Ca}^{2+}$ transients produced solely by the uptake of the extracellular $\mathrm{Ca}^{2+}$ (red and green) or solely by its discharge from the ER (blue). Each trace is an average for at least 15 cells.. Stimuli were applied at $60 \mathrm{~s}$; the intensities were adjusted to evoke similar responses. (B) Traces from the panel (A) were differentiated in order to emphasize the rate of $\mathrm{Ca}^{2+}$ increase. For clarity, the traces were filtered, and the premature onset of the response is a filtering artifact; see text for details. (C) Peak rates of $\mathrm{Ca}^{2+}$ increase as measured from individual traces without filtering; same data as in (A) and (B). (D) Peak rates of $\mathrm{Ca}^{2+}$ increase for $\mathrm{Ca}^{2+}$ transients evoked by 10- and 60-ns stimuli in the presence of $2 \mathrm{mM}$ external $\mathrm{Ca}^{2+}$ (the data from Fig. 2). Measurements were done without averaging or filtering.. Mean $+/-$ s.e, $n \geq 15 ; * \mathrm{p} \leq 0.01$. 